\title{
Study for License Plate Detection
}

\author{
Mie Mie Aung \\ University of Computer Studies (Monywa), Myanmar \\ Email: miemieaung781@gmail.com \\ Phyu Phyu Khaing \\ Myanmar Institute of Information Technology, Mandalay, Myanmar \\ Email: phyu_phyu_khaing@miit.edu.mm \\ Myint San \\ University of Computer Studies (Monywa), Myanmar \\ Email: myintsan013@gmail.com
}

Received: 15 September 2019; Accepted: 24 October 2019; Published: 08 December 2019

\begin{abstract}
License Plate Detection (LPD) system is the application of computer vision and image processing technology. LPD system is the first and main step of License Plate Recognition (LPR) system. So, it performs as the main driver of the LPR system. License plate detection step is always performed in front of the license plate recognition step. LPD system takes the vehicle images as input, follows with the general steps: such as reprocessing, localization, region extraction, and region detection, and the detected image are the output of the system. There are many algorithms for LPD while detecting a license plate in different conditions is still a complex task. For the LPD system, morphological operation and deep learning model are mostly used. This paper presents the critical study of the license plate detection system and also examines the implementation of new technologies of the license plate detection system.
\end{abstract}

Index Terms - License plate detection, image processing, edge detection algorithm, morphological operations, adaptive thresholding algorithm

\section{INTRODUCTION}

For the smart cities, intelligence transportation system is important for promoting the applications that used in the surveillance system of highway road, electronic toll gate system, traffic control system, and many others. License Plate Recognition (LPR) system is one of the main drivers of intelligent transportation systems. LPR recognizes the characters that exist over the license plates to identify the vehicle by applying image processing and computer vision techniques. LPR system composed of two main parts: License Plate Detection (LPD), and Character Segmentation and Recognition. LPD is the basic part of LPR system and the accuracy and the processing speed of LPR system are dependent on the accuracy of LPD and the efficiency of running time. So,
LPD system is the important step or the heart of LPR System.

The most common useful detection methods for license plate $[1,2,3,4,5]$ are edge detection, morphological operation, color and texture based detection, and deep learning based detection. License plates have many different characteristics [6]:

- Color: the type of the license plate can distinguish with the color of license plate. The type is mostly difference on the background color of license plate.

- Texture: the license plate has a continuous regular border, and edge features are more abundant in the vertical direction than the horizontal direction.

- Text: the number of license plate is composed of numbers and characters. Many cities' license plate is written with the English letters and numbers.

- Style: the style of license plate is different based on the shape, area, space of character and distance between the characters and borders.

The main objective of comparative research is the identification of similarities and differences between social entities. In our community, traffic must be monitored and vehicle registration can be achieved. LPD system serves to track incoming and exiting traffic by municipalities. The purpose of this study is to understand an effective, automated identification scheme for the approved car using the number plate for the car. It aims to draw conclusions about past events which may help to anticipate or explain future events.

This paper is organized with the following section. Section 2 introduces about the critical reviews that have been recently. Section 3 describes the comparison of previous methods. Section 4 presents the general steps of license plate detection system and section 5 finally concludes the paper and then follows the references 


\section{CRITICAL REviEWS}

Rabbani et al. proposed the License Plate Detection and Recognition System for Bangladeshi License by utilizing Morphological operation and convolutional neural network. Their system composed with four modules: license plate area detection, license plate extraction, characters and words segmentation, and characters and words recognition. Image preprocessing works image resizing, image enhancement by using haze removal techniques, and noise removal by applying wiener 2-D filter. The aspect ratio between 1.9 and 2.1 is used to detect the area of license plate. After detection, horizontal line rescaling and vertical line rescaling are used in the corners of license plate. Connected component analysis is used for character segmentation and convolutional neural network is used to recognize the character over license plate. The authors implemented the system on the customized dataset for digits and characters of Bangla. All accuracies of detecting license plate, segmenting and recognition the characters are over $90 \%$. The accuracy will be loss when the license plate's characters are separate. This system used small dataset, and need high resolution image to detect broken characters for the characters segmentation. [7]

Yuan et al. proposed the robust and efficient techniques for the real time detection of license plate in complex scenes by applying novel line density filter (LDF) for candidate extraction and cascaded license plate classifier (CLPC) for candidate verification. Downsampling and gray scale conversion of image are used in the pre-processing step. Before Line Density Filter for Candidate Extraction, edge detection and binarization are processed by using Sobel operator and adaptive thresholding (AT) respectively. After extracting the candidate region, finding the candidate with connected-component labeling (CCL) and removing the un-exhibited area of the license plate image is operating. Finally, the license plates are identified from among the candidate regions that detected from the image by applying a cascaded license plate classifier (CLCP) and used the saliency features of two color channels: HSV and RGB. The system is implemented on the two datasets: Caltech vehicle dataset and their collected PKU dataset. Average accuracy of the system is $96.62 \%$ and the average run time is less than other approaches. Different scenes are the limitation of the system and the authors suggested interesting the MSER or Hough transform approach for the further study. [8]

Character-specific extremal regions (ERs) and hybrid discriminative restricted Boltzmann machines (HDRBMs) are presented for a vehicle license plate recognition by Gou et al.. There are four main steps for vehicle license plate recognition: license plates' coarse detection, character region extraction, license plate detection, and license plate recognition. In the first step, background noises are removed with top-hat transformation, vertical edges are detected with Sobel filter, and back spaces are removed by applying Closing that is one of morphological operations. Real AdaBoost classifier is applied to select character-specific ERs as the region of characters in the second step. For the license plate detection step, license plate location and character segmentation are processed by inferring geometrical character attributes. In the last step, histogram of oriented gradients (HOG) descriptors is used to extract the features of character regions and HDRBM are used to recognize the character as an offline trained classifier. The system is experimented on the dataset extended the conference version dataset with real traffic monitoring scenes under various illumination conditions. The average performance percentages of License Plate Detection Rate (LDR), Character Recognition Rate (CRR), Overall Performance (OVR1 and OVR2) are 95.9, 98.2, 91.9 and 94.1, respectively. Further work may be deep architectures for location and recognition. [9]

Davis et al. introduced the faster license plate detection mechanism by using vertical edge detection algorithm (VEDA). The gray image conversion and adaptive thresholding are used in the preprocessing step. After preprocessing, vertical edges detection step is processed by applying unwanted lines estimation algorithm (ULEA) and vertical edge detection algorithm (VEDA). And then, License Plate Extraction are operated by implementing highlight desired details (HDD), candidate region extraction (CRE), plate region selection (PRS) and eliminating unwanted regions (EUR). For working in the global system, the system experiments on Indian vehicle image and also on different countries license plate images. The VEDA for vertical edge detection can more detect than histogram equalization and morphology operations. License plate detection system based on VEDA takes less computation time and it can detect other text blocks. However, it cannot detect in the highly blur image. [10]

Fomani and his colleague proposed the license plate detection algorithm by implementing adaptive morphological closing to locate the regions of gray level image, local adaptive thresholding for image smoothing, and morphological opening to separate the license plate region and other regions. Local histogram equalization (LHE) is used to preprocess the image before the main steps of the system. Adaptive morphological closing is composed of dilation, constructed structuring elements and erosion. Local adaptive thresholding is calculated based on the maximum and minimum value of width and height of license plate. The detection rate and computation time of the approach is measured on some real dataset collected in different situations. The detection rate of the algorithm is more than $99 \%$ and the computation time of the algorithm is faster than other algorithms. [11]

In 2017, Li et al. proposed a powerful license plate detection method by using Convolutional Neural Network (CNN). The system firstly generates the convolutional feature maps from the vehicle image using $\mathrm{CNN}$, and then complete license plate sub-window are extracted on the convolutional feature maps by applying a single-scale sliding-window detector. Finally, a regression network is used to locate the license plate. The system is implemented on their own high-quality data set. 
The CNN-based license plate detection method is more powerful than others and it can reduce the detection time. But, the image that takes on the illumination status is difficult to detect and the CNN-based detection method is needed to add the image enhancement methods. [12]

Shi et al. introduced the detection algorithm that applied the visual feature and convolutional neural network. The algorithm contains two main parts: candidate box generation, and candidate box classification and regression. For candidate box generation, there are two types: edge detection based method and color model based method. Edge detection based method is operated by using Gaussian blurring, grayscale conversion, sobel edge detection, binarization, and closing operation. In color model based method, HSV color space conversion, denoising using mean value, dilation and closing operation are processing on the color features. In the candidate box classification and regression step, two cascaded convolutional neural networks, namely C-Net and R-Net, are applied. C-Net is used to determine the place that exist the license plate and R-Net is used to judgment the position and size on the results of C-Net. The experiment is worked on the UCSD dataset and their own collected Chinese license plate images dataset. The algorithm that used visual features and convolutional neural network gets good performance on the given dataset, improved accuracy rate, and faster performance speed. However, the algorithm still has issues such as miss detection in actual scenes that contain many license plates and not satisfaction in the candidate box regression. [13]

The comparison of the previous research works is presented in this section with two tables. This section is aimed to know about the methods and datasets that used in the previous study and the advantage and disadvantages of the previous studies. Table 1 shows the comparison of the license plate detection methods and the datasets that used in the reference papers. Table 2 presents the advantages and disadvantages of the license plate detection methods

Table 1. Detection methods and Lecense Plate Dataset

\begin{tabular}{|c|c|c|}
\hline References & Detection Methods & Datasets \\
\hline Y. Yuan et al. (2017) & $\begin{array}{l}\text { - Sobel Edge Detection } \\
\text { - Adaptive Thresholding } \\
\text { - Line Density Filter } \\
\text { - Connected-Component Labeling } \\
\text { - Cascaded License Plate Classifier }\end{array}$ & $\begin{array}{l}\text { - Caltech vehicle dataset } \\
\text { - PKU dataset. }\end{array}$ \\
\hline Davis et al. (2015) & $\begin{array}{l}\text { - Unwanted Lines Estimation Algorithm } \\
\text { - Vertical Edge Detection Algorithm } \\
\text { - Highlight Desired Details } \\
\text { - Candidate Region Extraction } \\
\text { - Plate Region Selection } \\
\text { - Eliminating Unwanted Regions }\end{array}$ & $\begin{array}{l}\text { - Indian vehicle image } \\
\text { - Different countries license plate images }\end{array}$ \\
\hline $\begin{array}{l}\text { Fomani et al. } \\
(2017)\end{array}$ & $\begin{array}{l}\text { - Adaptive Morphological Closing } \\
\text { - Local Adaptive Thresholding } \\
\text { - Morphological Opening }\end{array}$ & - Real dataset collected in different situations \\
\hline $\begin{array}{l}\text { Li et al. } \\
(2017)\end{array}$ & $\begin{array}{l}\text { - Convolutional Neural Network } \\
\text { - Single-Scale Sliding-Window Detector } \\
\text { - Regression Network }\end{array}$ & - Their own created high quality dataset \\
\hline $\begin{array}{l}\text { Shi et al. } \\
(2018)\end{array}$ & $\begin{array}{l}\text { - Sobel Edge Detection } \\
\text { - Morphological Operation } \\
\text { - Convolutional Neural Network } \\
\text { (C-Net and R-Net) }\end{array}$ & $\begin{array}{l}\text {-UCSD dataset } \\
\text { - Their own collected Chinese license plate } \\
\text { images dataset }\end{array}$ \\
\hline
\end{tabular}

Table 2. Advantages and disadvantages of license plate detection

\begin{tabular}{|l|l|l|}
\hline References & Advantages & Disadvantages \\
\hline Y. Yuan et al. (2017) & $\begin{array}{l}\text { • Increase detection ratio } \\
\bullet \text { Decrease the run time }\end{array}$ & $\bullet$ Fails to detect the reflective glare image \\
\hline Davis et al. (2015) & $\begin{array}{l}\text { - Less } \\
\text { time } \\
\text { Detect license plate even there is other text } \\
\text { blocks are present }\end{array}$ & $\bullet$ Fails to detect highly blurred image \\
\hline
\end{tabular}




\begin{tabular}{|l|l|l|}
\hline $\begin{array}{l}\text { Fomani et al. } \\
(2017)\end{array}$ & $\begin{array}{l}\text { - High detection rate } \\
\text { - Less computation time }\end{array}$ & $\begin{array}{l}\bullet \text { Fails to detect the license plate at } \\
\text { insignificant regions }\end{array}$ \\
\hline $\begin{array}{l}\text { Li et al. } \\
(2017)\end{array}$ & $\begin{array}{l}\text { - Powerful detection rate } \\
\text { - Less detection time }\end{array}$ & $\begin{array}{l}\bullet \text { Problems on the high illuminated image } \\
\bullet \text { Needs to add image enhancement method }\end{array}$ \\
\hline $\begin{array}{l}\text { Shi et al. } \\
(2018)\end{array}$ & $\begin{array}{l}\text { - Good performance on the given dataset, } \\
\text { - Faster performance speed }\end{array}$ & $\begin{array}{l}\bullet \text { Miss detection in actual } \\
\text { Not satisfaction in the candidate box } \\
\text { regression }\end{array}$ \\
\hline
\end{tabular}

\section{Steps For Licens Plate Detection}

License plate detection can be implemented to have set of robust methods for each of following steps:
a. Image Pre-processing
b. Image Localization
c. License Plate Region Extraction
d. License Plate Region Detection

For all of above steps, the set of suitable methods or robust method can be designed and used for detection the license plate. For image pre-processing, image resizing, image enhancement, and color transformation are mostly processed. For detection, morphological operations, edge detection algorithms, and thresholding algorithms are mostly applied. The general steps are illustrated in Figure 1.

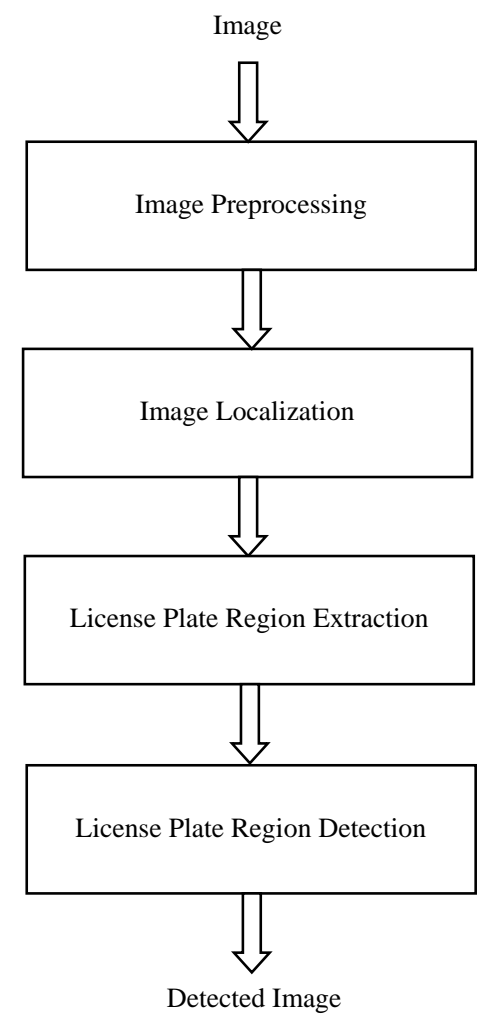

Fig.1. General steps for license plate detection

\section{Detection Algorithms}

\subsection{Edge Detection Algorithms}

Edge detection is an image processing technique for finding the boundaries of objects with images. It works by detecting discontinuities in brightness. Edge detection is used for image segmentation and data extraction. There are many popular edge detection algorithms. Among them, this will discuss the mostly used for license plate detection, such as sobel edge detection, canny edge detection, and prewitt edge detection.

\subsubsection{Sobel Edge Detection}

Sobel edge detection is one of the most popular edge detection techniques. It calculates the gradient intensity at each pixel of the image and finds the direction changing rate that is the largest increase from brightness to darkness. It uses two kernels as the sobel filter. One filter is used to change the horizontal direction and another one is used to change the vertical direction. Two kernels $\left(G_{x}\right.$ and $G_{y}$ ) are convolved with the original images $(A)$ to detect the horizontal and vertical edges as shown in equation 1 and 2 ; the gradient of image is calculated as equation 3; and figure 2 shows the sobel edge detection result.

$$
\begin{gathered}
G_{x}=\left[\begin{array}{ccc}
-1 & 0 & +1 \\
-2 & 0 & +2 \\
-1 & 0 & +1
\end{array}\right] * A \\
G_{y}=\left[\begin{array}{ccc}
+1 & +2 & +1 \\
0 & 0 & 0 \\
-1 & -2 & -1
\end{array}\right] * A \\
G=\sqrt{G_{x}^{2}+G_{y}^{2}}
\end{gathered}
$$

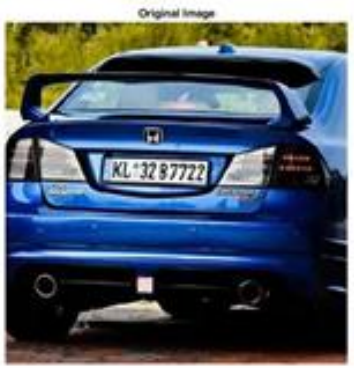

(a)

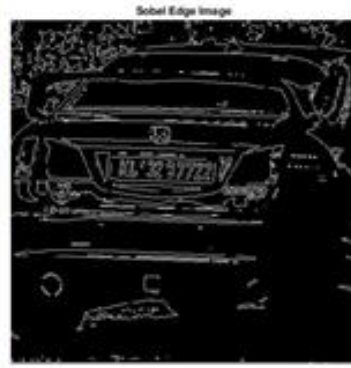

(b)
Fig.2. (a) Original RGB Image, (b) Results of Sobel Edge Detection 


\subsubsection{Canny Edge Detection}

Canny edge detection is a multi-stage edge detection algorithm that used in the detection of wide range of edges in images. The canny edge detection algorithm is composed with 7 steps:
a. Grayscale Conversion
b. Noise Removal using Gaussian Blurring
c. Intensity Gradient Calculating
d. Non-maximum Suppression
e. Double Thresholding
f. Edge Tracking by Hysteresis
g. Weak Edges Cleaning

The first step converts the original image into the grayscale image with the range of pixel values from 0 to 255; and then, the Gaussian blurring is performed on the image to remove the noise. After that, the intensity gradient is calculated with sobel filter by using equations 1,2 and 3 . In step 4, non-maximum suppression is applied to thin the edges of the image by finding the pixel with the maximum value in an edge. Non maximum suppression can be achieved by interpolating the pixels as equation 4 for greater accuracy.

$$
r=\alpha b+(1-\alpha) a
$$

In double thredsholding step, strong, weak and nonrelevant pixels are identified. Strong pixels are high intensity pixels; weak pixels are pixels that the intensity value is not reach to strong; and non-relevant pixels are pixels that have small intensity values. High threshold is used to identify the strong pixels with equation 5 and low threshold is used to identify the non-relevnat pixels with equation 6 .

highThreshold $=(\max ($ image $)) *$ highThresholdRatio

lowThreshold=highThreshold*lowThresholdRatio

After that, hysteresis for edge tracking is to determine the strong edges and weak edges. The weak edges that connected with strong edges are determined as the actual edges and weak edges that do not connect with strong edges will be removed. It works with recursively iterate. Finally, the remaining weak edges set with zero through the iteration. The result of canny edge detection of image is shown in figure 3 .

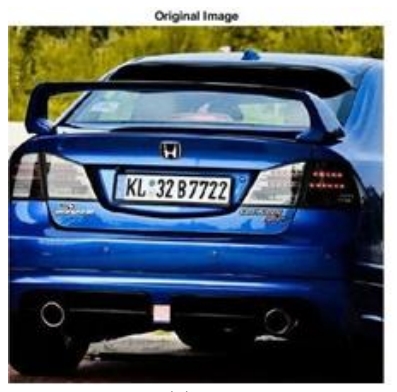

(a)

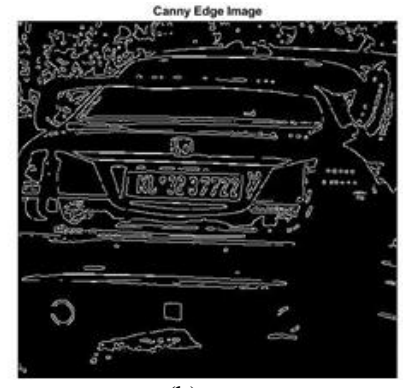

(b)
Fig.3. (a) Original RGB Image, (b) Results of Canny Edge Detection

\subsubsection{Prewitt Edge Detection}

Prewitt edge detection is similar with sobel edge detection but Prewitt operator is used for vertical and horizontal edge detection of an image. Vertical and horizontal edges are calculated by using difference two derivative masks $\left(G_{x}\right.$ and $\left.G_{y}\right) \cdot G_{x}$ is used for vertical edge detection and $\mathrm{G}_{\mathrm{y}}$ is used for the horizontal edge detection. Difference two derivatives masks have three properties.

- opposite sign should be present in the mask.

- sum of mask should be equal to zero.

- more weight means more edge detection.

\begin{tabular}{|l|l|l|}
\hline-1 & 0 & 1 \\
\hline-1 & 0 & 1 \\
\hline-1 & 0 & 1 \\
\hline \multicolumn{3}{|c|}{$\mathrm{G}_{\mathrm{x}}$}
\end{tabular}

\begin{tabular}{|c|c|c|}
\hline-1 & -1 & -1 \\
\hline 0 & 0 & 0 \\
\hline 1 & 1 & 1 \\
\hline \multicolumn{3}{|c|}{$\mathrm{G}_{\mathrm{y}}$}
\end{tabular}

Figure 4 and 5 show the results that is the prewitt edge detection by applying vertical mask and horizontal mask respectively.

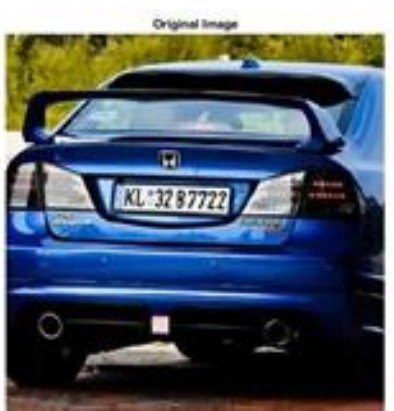

(a)

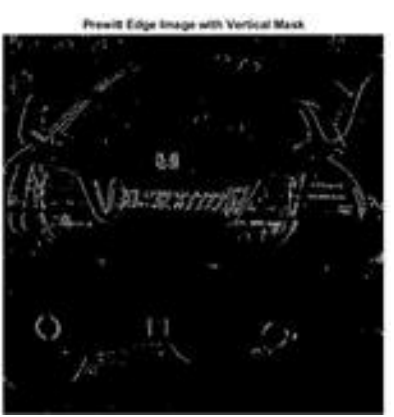

(b)
Fig.4. (a) Original RGB Image, (b) Results of Prewitt Edge Detection with Vertical Mask

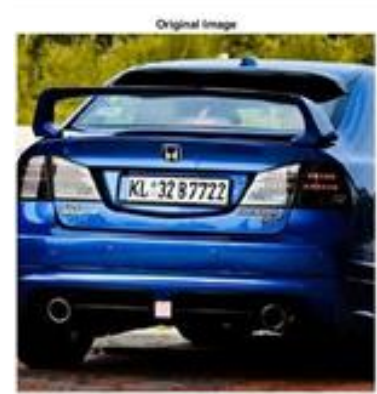

(a)

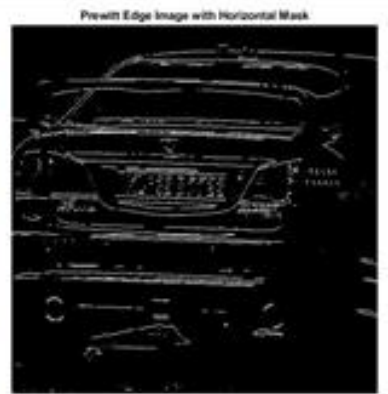

(b)
Fig.5. (a) Original RGB Image, (b) Results of Prewitt Edge Detection with Horizontal Mask

\subsection{Morphological Operations}

Morphological is an image processing operation that processes based on the shape of the image. Morphological operations are used to extract the components of image and there are commonly used to represent and describe the region shape of the image. 
Morphological operations apply a structuring element to an input image, creating an output image of the same size. The morphological operations are based on the set theory of the mathematical operations and that operations are powerful for the image processing. There are many morphological operations in image processing such as opening, closing, erosion, dilation, and many others. $3 * 3$ square structuring elements are applied for all experiments.

\subsubsection{Opening}

Opening is used to take smoothing contours, breaking the necks, and eliminating protrusions. Opening is firstly worked the erosion and follows by the dilation. Opening two objects $\mathrm{X}$ and $\mathrm{Y}$ is showed in equation 7 and sample results are shown in figure 6.

$$
X \circ Y=(X \Theta Y) \oplus Y
$$

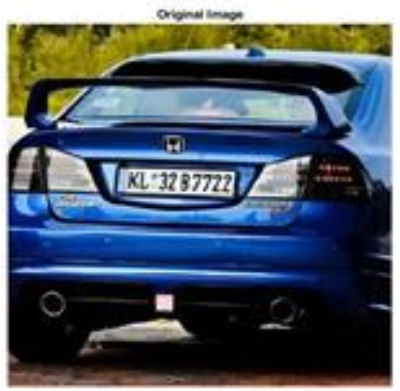

(a)

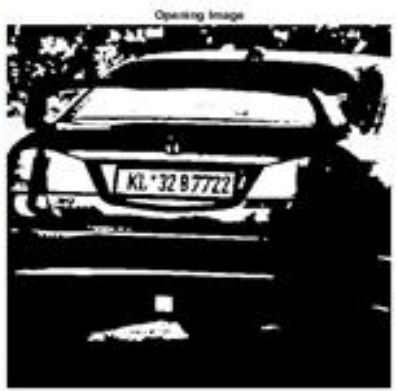

(b)
Fig.6. (a) Original RGB Image, (b) Opening Image

\subsubsection{Closing}

Closing is used to take smoothing the sections of contours, fusing the narrow or long thin gulfs, eliminating the small holes, and filling in the contour gaps. Closing is firstly dilated and then eroded the results. Equation 8 shows the closing two objects $\mathrm{X}$ and $\mathrm{Y}$, and figure 7 shows the sample results of closing operation.

$$
X \bullet Y=(X \oplus Y) \Theta Y
$$

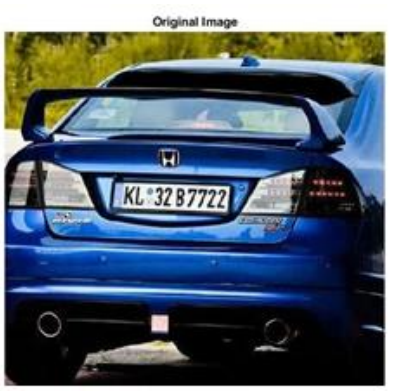

(a)

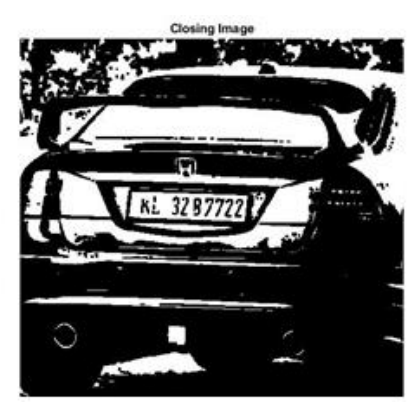

(b)
Fig.7. (a) Original RGB Image, (b) Closing Image

\subsubsection{Erosion}

Erosion is used to shrink the one object by using other object. Morphological erosion removes islands and small objects so that only substantive objects remain. It removes the boundary pixels of the object. The value of the output pixel is the minimum value of all pixels in the neighborhood. In a binary image, a pixel is set to 0 if any of the neighboring pixels have the value 0 . Erosion is applied for the application that eliminates the irrelevant details from the binary image. Figure 8 shows the comparison of the original RGB image and erosion image.

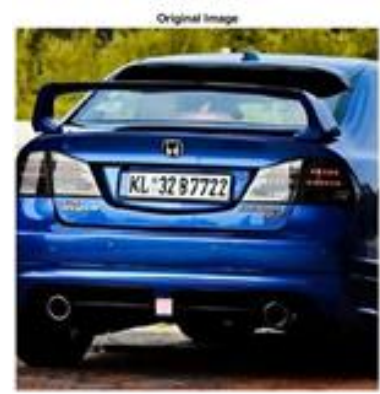

(a)

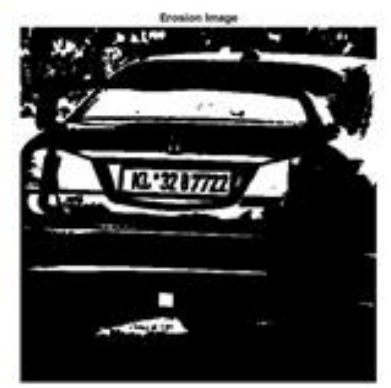

(b)
Fig.8. (a) Original RGB Image, (b) Erosion Image

\subsubsection{Dilation}

Dilation is used to expand the one object by using the structuring another object. Morphological dilation makes objects more visible and fills in small holes in objects. It adds the pixels at the boundaries of the image. The value of the output pixel is the maximum value of all pixels in the neighborhood. In a binary image, a pixel is set to 1 if any of the neighboring pixels have the value. When the gaps are bridging, dilation is applied as the simplest applications. Figure 9 shows the comparison of the original RGB image and dilation image.

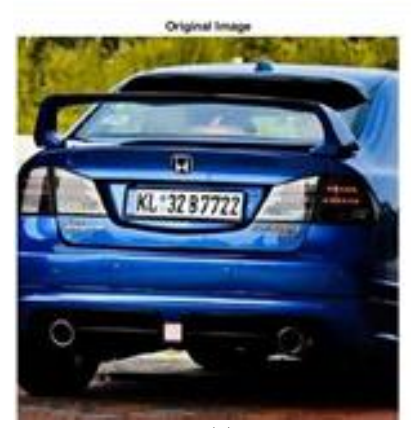

(a)

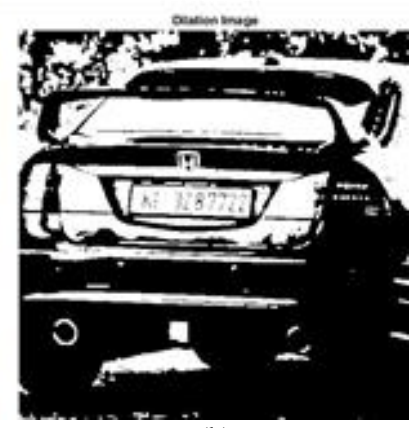

(b)
Fig.9. (a) Original RGB Image, (b) Dilation Image

\subsection{Adaptive Thresholding Algorithm}

An adaptive thresholding algorithm separates the foreground from the background with nonuniform illumination. Adaptive Thresholding (AT) [14] is exploited to eliminate weak edges and generate a binary edge image. For the edge detection, AT performs to enhance the estimated edges and generate a binary edge image. The AT method generates an integral image by summing all pixel values from the upper left corner for each pixel of given image. Then, a binary edge image is generated by thresholding each pixel in the integral image using a threshold that is adaptively computed from a local window in integral image [14]. 


\section{CONCLUSION}

This paper has studied few important research works of the license plate detection system. For license plate detection systems, the input is the vehicle images and the output is the detected images. License plate detection systems are mostly used morphological operation and deep learning model. The detection methods and the datasets are summarized, and the advantages and disadvantages of license plate detection methods are also presented in this paper. This paper discusses the mostly useful detection methods and also shows the results of detection methods that implement on the MATLAB. The general steps are also describes that mostly used for license plate detection.

\section{ACKNOWLEDGMENT}

We would like to give our thanks and gratitude to people who have contributed towards the development of this manuscript from the planning stage to the finish stage.

\section{REFERENCES}

[1] Shi J, Malik J., "Normalized Cuts and Image Segmentation", IEEE Computer Society, 2000.

[2] Felzenszwalb P F, Huttenlocher D P., "Efficient GraphBased Image Segmentation", International Journal of Computer Vision, 2004, 59(2):167-181.

[3] Pal N R., "A review on image segmentation techniques", Pattern Recognit, 1993, 26(9):1277-1294.

[4] Boykov Y., "Interactive graph cuts for optimal boundary and region segmentation of object in N-D image segmentation", In Proceedings eighth IEEE international conference on computer vision., ICCV 2001, Vol. 1, pp. $105-112$.

[5] Z. K. Huang, and L. Y. Hou, "Chinese License Plate Detection Based on Deep Neural Network", International Conference on Control and Robots (ICCR)(pp. 84-88), 2018.

[6] Mao, Shangqin, X. Huang, and M. Wang., "An adaptive method for Chinese license plate location", Intelligent Control and Automation IEEE, 2010:6173-6177.

[7] G. Rabbani, M.A. Islam, M.A. Azim, M.K. Islam, and Md.M. Rahman, "Bangladeshi License Plate Detection and Recognition with Morphological Operation and Convolution Neural Network", $201821^{\text {st }}$ International Conference of Computer and Information Technology (ICCIT), pp. 1-5. IEEE, 2018.

[8] Y. Yuan, W.Zou, Y. Zhao, X. Wang X. Hu, and N. Komodakis, "A Robust and Efficient Approach to License Plate Detection", IEEE Transactions on Image Processing, 26(3), pp. 1102-1114, 2017.

[9] Chao Gou, Kunfeng Wang, Yanjie Yao, and Zhengxi Li, "Vehicle License Plate Recognition Based on Extremal Regions and Restricted Boltzmann Machines", IEEE Transactions on Intelligent Transportation Systems, 17(4), pp.1096-1107, 2015.

[10] Davis, A. M., Arunvinodh, C., and Np, A. M. , "Automatic license plate detection using vertical edge detection method.", In 2015 International Conference on Innovations in Information, Embedded and Communication Systems (ICIIECS) (pp. 1-6), 2015, March, IEEE.
[11] Babak Abad Fomani, and Asadollah Shahbahrami, "License plate detection using adaptive morphological closing and local adaptive thresholding", 3rd International Conference on Pattern Recognition and Image Analysis (IPRIA), pp. 146-150, 2017.

[12] H. Li, R. Yang, and X. Chen, "License plate detection using convolutional neural network", 3rd International Conference on Computer and Communication, pp. 1736$1740,2017$.

[13] Y. Shi, and Y. Chen, "License plate detection based on convolutional neural network and visual feature", International Conference on Mechanical, Control and Computer Engineering (ICMCCE), pp. 514-519, 2018.

[14] D. Bradley and G. Roth, "Adaptive thresholding using the integral image," J. Graph., GPU, Game Tools, vol. 12, no. 2, pp. 13-21, 2007.

\section{Authors' Profiles}

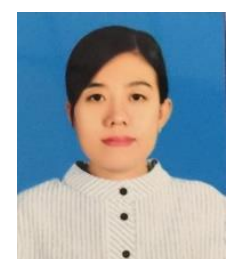

Mie Mie Aung: Lecturer of Faculty of Information Science in Computer University (Monywa), Myanmar. She completed her B.C.Sc. and B.C.Sc. (Hons.) degrees from Computer University (Monywa), Myanmar in 2004 and 2005 respectively. She got M.C.Sc. degree from University of Computer Studies, Mandalay, Myanmar in 2007. She interested in image processing, and data mining.

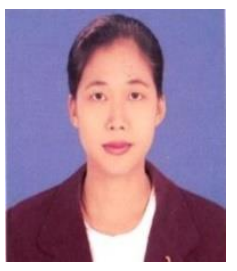

Phyu Phyu Khaing: Assistant Lecturer in the Myanmar Institute of Information Technology, Mandalay, Myanmar. She received her B.C.Sc. degree in 2007, B.C.Sc. (Hons.) in 2008 and M.C.Sc. degree in 2010 from University of Computer Studies (Monywa), Myanmar. She is currently working toward the Ph.D. degree in University of Computer Studies, Mandalay, Myanmar. Her research interests include computer vision, digital image processing, and Deep Learning.

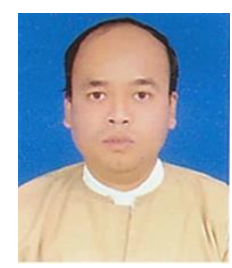

Myint San: Lecturer of Faculty of Information Science in Computer University (Monywa), Myanmar. He received his B.C.Sc degree in 2003, and B.C.Sc(Hons;) in 2004 from University of Computer studies (Monywa), Myanmar. He graduated for M.C.Sc degree in 2005 from University of Computer Studies, Mandalay, Myanmar. His research interest includes image processing, and data mining. 
How to cite this paper: Mie Mie Aung, Phyu Phyu Khaing, Myint San, " Study for License Plate Detection", International Journal of Image, Graphics and Signal Processing(IJIGSP), Vol.11, No.12, pp. 39-46, 2019.DOI: 10.5815/ijigsp.2019.12.05 